\title{
Reversibly switchable photoacoustic tomography using a genetically encoded near-infrared phytochrome
}

Junjie Yao, Andrii A. Kaberniuk, Lei Li, Daria M. Shcherbakova, Ruiying Zhang, et al.

Junjie Yao, Andrii A. Kaberniuk, Lei Li, Daria M. Shcherbakova, Ruiying Zhang, Lidai Wang, Guo Li, Vladislav V. Verkhusha, Lihong V. Wang, "Reversibly switchable photoacoustic tomography using a genetically encoded near-infrared phytochrome," Proc. SPIE 9708, Photons Plus Ultrasound: Imaging and Sensing 2016, 97082 U (18 March 2016); doi: $10.1117 / 12.2229156$

SPIE. Event: SPIE BiOS, 2016, San Francisco, California, United States 


\title{
Reversibly switchable photoacoustic tomography using a genetically- encoded near-infrared phytochrome
}

\author{
Junjie Yao ${ }^{\mathrm{a}}$, Andrii A. Kaberniuk ${ }^{\mathrm{b}}$, Lei Li ${ }^{\mathrm{a}}$, Daria M. Shcherbakova ${ }^{\mathrm{b}}$, Ruiying Zhang ${ }^{\mathrm{a}}$, Lidai Wang ${ }^{\mathrm{a}}$, \\ Guo Li ${ }^{\mathrm{a}}$, Vladislav V. Verkhusha ${ }^{\mathrm{b}, \mathrm{c}^{*}}$ and Lihong V. Wang ${ }^{\mathrm{a}^{*}}$ \\ ${ }^{a}$ Department of Biomedical Engineering, Washington University in St. Louis, \\ St. Louis, MO 63130, USA, \\ ${ }^{b}$ Department of Anatomy and Structural Biology and Gruss-Lipper Biophotonics Center, Albert \\ Einstein College of Medicine, Bronx, NY 10461, USA, \\ ${ }^{\mathrm{c}}$ Department of Biochemistry and Developmental Biology, Faculty of Medicine, University of \\ Helsinki, Helsinki 00290, Finland
}

"Correspondence should be addressed to: V.V.V. (vladislav.verkhusha@einstein.yu.edu) and L.V.W. (lhwang@wustl.edu)

\begin{abstract}
Optical imaging of genetically encoded probes has revolutionized biomedical studies by providing valuable information about targeted biological processes. Here, we report a novel imaging technique, termed reversibly switchable photoacoustic tomography (RS-PAT), which exhibits large penetration depth, high detection sensitivity, and super-resolution. RS-PAT combines advanced photoacoustic imaging techniques with, for the first time, a nonfluorescent photoswitchable bacterial phytochrome. This bacterial phytochrome is the most near-infrared shifted genetically encoded probe reported so far. Moreover, this bacterial phytochrome is reversibly photoconvertible between its far-red and near-infrared light absorption states. Taking maximum advantage of the powerful imaging capability of PAT and the unique photochemical properties of the phytochrome, RS-PAT has broken through both the optical diffusion limit for deep-tissue imaging and the optical diffraction limit for super-resolution photoacoustic microscopy. Specifically, with RS-PAT we have achieved an unprecedented detection sensitivity of $\sim 2 \mu \mathrm{M}$, or as few as $\sim 20$ tumor cells, at a centimeter depth. Such high sensitivity is fully demonstrated in our study by monitoring tumor growth and metastasis at whole-body level with $\sim 100 \mu \mathrm{m}$ resolution. Moreover, our microscopic implementation of RS-PAT is capable of imaging mammalian cells with a sub-diffraction lateral resolution of $\sim 140 \mathrm{~nm}$ and axial resolution of $\sim 400 \mathrm{~nm}$, which are respectively $\sim 2$-fold and $\sim 75$-fold finer than those of our conventional photoacoustic microscopy. Overall, RS-PAT is a new and promising imaging technology for studying biological processes at different length scales.
\end{abstract}

\section{KEYWORDS}

Photoacoustic tomography; Photoacoustic microscopy; Genetic imaging; Super-resolution imaging; Deep tissue imaging; Reversibly switchable protein; Bacterial phytochrome; Near-infrared protein;

\section{INTRODUCTION}

Taking advantage of various optical contrasts in biological tissues, optical imaging techniques have provided valuable information for biomedical studies at different length scales [1]. However, strong optical scattering in tissue has led to substantial tradeoff between the spatial resolution and penetration depth. Photoacoustic (PA) tomography (PAT), on the contrast, has overcome the depth and resolution limitations of pure optical imaging, by acoustically detecting the optical absorption contrast [2]. PAT is inherently suited for molecular imaging, with a wealth of exogenous contrasts, such as organic dyes, nanoparticles, and fluorescent proteins [3]. However, systematically-administered organic dyes and nanoparticles present challenges in effective targeting and clearing [4-6]. Existing fluorescent proteins lack strong optical absorption at wavelengths beyond $750 \mathrm{~nm}$, and thus are not suited for deep tissue imaging [7, 8]. Genetic encoding of eumelanin has also been explored in PAT [9, 10]. However, the heavy-burden pigmentation may jeopardize the cell growth, and that the endogenous melanin in tissue may degrade the imaging specificity. In addition, all the above contrasts sufferer from the strong background signals generated by various endogenous biomolecules, especially hemoglobin, which typically have wideband absorption spectra and are much more abundant in tissue $[7,8,11]$. The

Photons Plus Ultrasound: Imaging and Sensing 2016, edited by Alexander A. Oraevsky, Lihong V. Wang Proc. of SPIE Vol. 9708, 97082U · (c) 2016 SPIE · CCC code: 1605-7422/16/\$18 - doi: 10.1117/12.2229156 
highly wavelength-dependent light attenuation in tissue prevents spectroscopic unmixing at depths beyond the diffusion limit [12].

Given these limitations, we develop a new approach that combines deep-penetration PA imaging with a novel nonfluorescent bacteri-phytochrome BphP1, allowing high-resolution ultra-sensitive imaging of the genetically encoded biological process at depths. By photoconverting between the red-light-absorption Pr state and the NIR-light-absorption Pfr state, BphP1 is a natural reversible switch in light perception [13, 14]. Capitalizing on the strong NIR absorption of BphP1 and its switching capability between two states, we demonstrate that this method can significantly enhance the detection sensitivity of PAT. Tens of mammalian cells can be detected at $\sim 1 \mathrm{~cm}$ depth. We further demonstrate this high detection sensitivity by imaging the growth of BphP1-expressing cancer cells in deep tissue in vivo and monitoring the cancer metastasis over an extended period of time. We also extended this method to optical-resolution PA microscopy, demonstrating substantially enhanced spatial resolution and image contrast on single bacteria, mammalian cells and mice.

\section{RESULTS}

\subsection{Synthesis and characterization of BphP1}

Among phytochromes, BphP1 is one of the most attractive sources for PAT contrast because its absorption spectrum is the most red- shifted and because it utilizes biliverdin IV $\alpha(\mathrm{BV})$ as a chromophore. BphP1 can be effectively switched between the Pr-state and Pfr-state by light illumination at $630 \mathrm{~nm}$ and $780 \mathrm{~nm}$, respectively. Biochemically, it is the bilin chromophore of BphP1 that absorbs light and changes the conformation of the attached protein, resulting in the switching from one state to the other (Figure 1a). Compared with other switchable photoreceptors that absorbs blue/ultra-violet light, including cryptochromes and phototropins, BphP1 is more suited for deep tissue imaging, with red-shifted absorption. BV is an intermediate of heme metabolism and is ubiquitously found in mammalian tissues, so BphP1 can be readily transfected in most biological systems [15]. However, because of its lack of fluorescence, wildtype BphP1 has never been used in optical imaging [13].

(a)

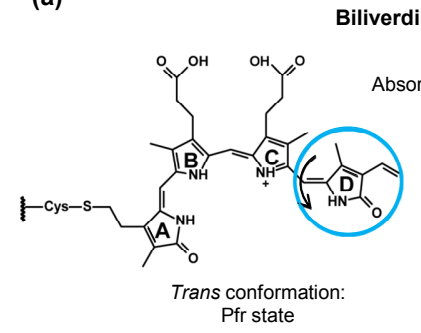

(c)

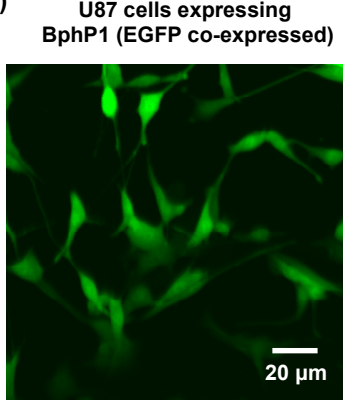

(d)

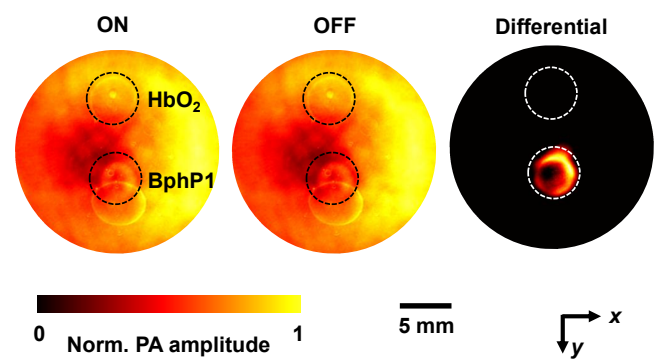

(b)

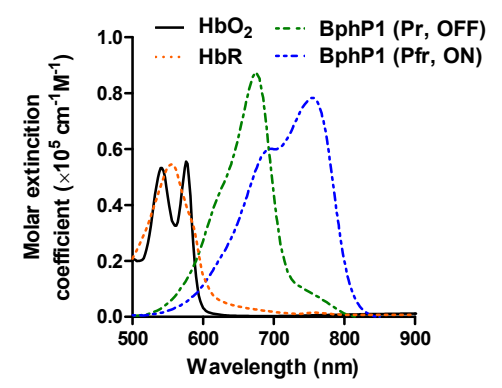

(e)

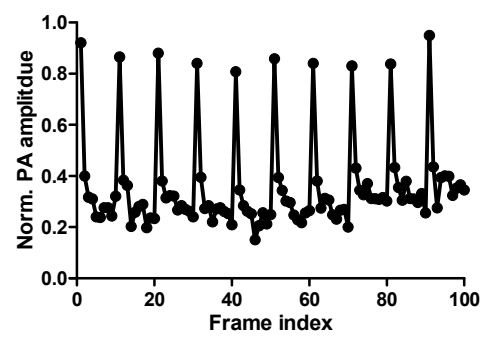

Figure 1. Reversibly switchable BphP1 as a non-fluorescent genetically-encoded photoacoustic imaging contrast. (a) Photoswitching of BphP1 from Pr state to Pfr state, and vice versa, with the NIR and far-red illumination. (b) Absorption spectra of oxy-hemoglobin, deoxy-hemoglobin, Pr and Pfr state BhpP1, showing the strong NIR absorption of BphP1. (c) U87 cells expressing BphP1, with EGFP co-expressed for fluorescence imaging. (d) The PA images of an optically scattering and absorbing phantom with BphP1-expressing U87 cells embedded at $10 \mathrm{~mm}$. The ON and OFF state images do not show the tumor cells due to the strong background signal, and the differential image clearly reveals the tumor cell signals. (e) Reversibly switching BphP1-expressing U87 cells in scattering media. 
We investigated BphP1 in deep tissue imaging. Plastic tubes filled with protein or blood were embedded at different depths in scattering media, and imaged by PAT at their peak absorption wavelengths. The molar extinction coefficient of Pr-state BphP1 at $630 \mathrm{~nm}$ is $\sim 40$ times that of oxy-hemoglobin $\left(\mathrm{HbO}_{2}\right)$, and Pfr-state BphP1 at $780 \mathrm{~nm}$ is $\sim 70$ times that of $\mathrm{HbO}_{2}$. We investigated $\mathrm{BphP1}$ as a potential PA contrast, by using an upgraded whole-body PAT system (Figure 1b) [16]. To cover the absorption spectra of all the above proteins, an optical parametric oscillator (OPO) laser and a Ti:Sapphire laser were synchronized to provide an excitation spectrum from $400 \mathrm{~nm}$ to $800 \mathrm{~nm}$. The signal amplitude at $10 \mathrm{~mm}$ depth dropped by $\sim 30 \mathrm{~dB}$ for $\mathrm{BphP} 1$ at $780 \mathrm{~nm}$. Therefore, $\mathrm{BphP} 1$ is more suited for deep tissue imaging due to the weaker light attenuation at longer wavelength. We quantified the noise equivalent detection concentrations (NEC) of the proteins at different depths. An NEC of $2.7 \mu \mathrm{M}$ has been achieved for BphP1 at $10 \mathrm{~mm}$ with a laser fluence of 8 $\mathrm{mJ} / \mathrm{cm}^{2}$, two orders of magnitude better than that of reported genetically encoded eumelanin at $600 \mathrm{~nm}[9]$.

We investigated the reversible switching of BphP1 between Pr- and Pfr-states, by measuring its optical absorbance at $780 \mathrm{~nm}$. We observed an exponential recovery from Pr-state to Pfr-state under $630 \mathrm{~nm}$ illumination, and an exponential decay from Pfr-state to Pr-state under $780 \mathrm{~nm}$ illumination. Such robust switching of BphP1 has allowed a simple differential operation on the PA images before and after the switching. The differential image can therefore suppress the background signal and enhance the image contrast [20]. Since hemoglobin is the dominant endogenous contrast in the visible-NIR spectral range, we further quantified the signal (protein) to background (blood) ratio at $10 \mathrm{~mm}$ depth. The results show that the differential image has substantially enhanced the contrast to noise ratio of BphP1 by $\sim 25$ times (Figure 1c). Multiple switching cycles did not cause photobleaching. The switching rate from Pf-state to Pfr-state is approximately proportional to the illumination light intensity at $630 \mathrm{~nm}$, similar to other switchable chromophores [1719]. We observed robust switching of BphP1 at $10 \mathrm{~mm}$ depth without causing photobleaching (Figure 1d).

\subsection{In vivo high-sensitivity PAT of mammalian cells expressing BphP1}

BphP1 can be readily expressed in mammalian cells, requiring no enzymes or exogenous cofactors. Here, we permanently transfected U87 glioblastoma cells with BphP1 gene and EGFP gene. The average BphP1 expression concentration in U87 cells was estimated to be $\sim 5 \mu \mathrm{M}$. Because BphP1 is non-fluorescent, EGFP is used for cell sorting and selection by fluorescence cytometry, and is also used for ex vivo validation of the in vivo PA imaging.
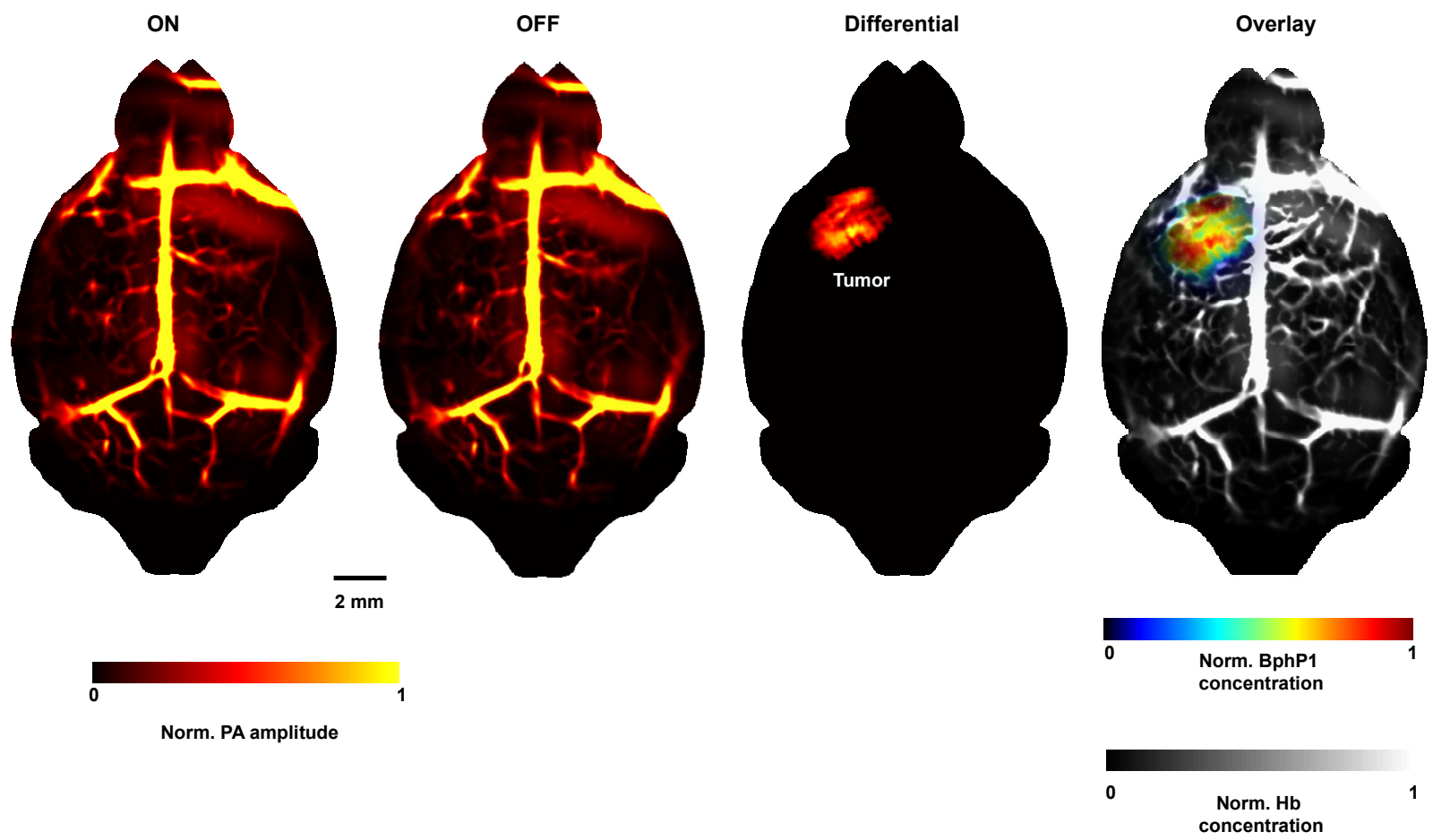

Figure 2. Deep PA imaging of a mouse brain tumor expressing BphP1. The ON and OFF state PA images of a nude mouse brain were acquired two weeks after the injection of U87 tumor cells at $\sim 3 \mathrm{~mm}$ underneath the surface. The tumor is invisible in either the ON state or OFF state images due to the overwhelming background signals from blood. The differential image, by contrast, clearly shows the tumor by substantially suppressing background signals. The overlay image shows the BphP1 signal in color and the background blood signal in gray. 
We investigated BphP1 for genetic encoded PA imaging in vivo. One million BphP1-expressing U87 cells were injected into the brain of a nude mouse, with the guidance of ultrasound imaging. Whole-body PA images were acquired one week after the inoculation of tumor cells (Figure 2). The BphP1-expressing U87 tumor in the brain could not be visualized in both the ON- and OFF-state PA images, due to the overwhelming blood signals. By contrast, the differential image clearly shows the tumor, with an average tumor-to-background ratio of $\sim 20$. While the tumor region has different signal amplitudes in the ON- and OFF-state images, the non-switchable background signals are identical. We harvested the brain and the histology confirmed the tumor region with higher cell density. We also demonstrated the three-dimensional imaging capability of PAT by stepping along the truck of a nude mouse bearing a BphP1-experessing U87 tumor in the flank. Although the elevational sectioning of current version PAT $(\sim 1 \mathrm{~mm})$ is worse than its in-plane resolution $(\sim 100 \mu \mathrm{m})$, it can be improved by using three-dimensional imaging reconstruction and high frequency ultrasound detection [21].

\subsection{Switchable photoacoustic microscopy with sub-diffraction resolution}

High scalability in both acoustic and optical domains is one of the most important advantages of PAT, allowing multiscale molecular imaging in vivo with the same optical contrast [2]. By focusing the excitation light, PAT has achieved optically-determined spatial resolution on the scale of micrometers and even submicrometers, within the optical diffusion limit. Here, we demonstrated that BphP1 can be used for optical-resolution PA imaging in vivo and for subdiffraction PA imaging of bacteria and mammalian cells, expanding genetically encoded PA imaging into cellular and subcellular domains.

We demonstrated a sub-diffraction PA imaging method by using BphP1. This approach, termed switchable photoacoustic microscopy (SPAM), is based on the switching dynamics of BphP1. When a Gaussian-shape $780 \mathrm{~nm}$ light strikes ON-state Bphp1 molecules, the generated PA signal is a summation of the contributions from all molecules inside the excitation volume. During the excitation, the BphP1 molecules inside the excitation volume are inhomogeneously switched OFF, at a rate proportional to the local excitation intensity. Therefore, the PA signal from the center of the excitation volume decays faster than that from the periphery. In the polynomial fitting of the resultant PA signal decay, the high order coefficient highlights the signal contribution from the center of the excitation volume, and thus sharpens the resolution in all dimensions (Figure 3a) [22]. More importantly, SPAM can greatly block the out-of-focus signals for large targets, and thus enhance the image contrast. Notably, since BphP1 can be robustly cycled between the ON and OFF states, the whole imaging process is essentially lossless.

(a)

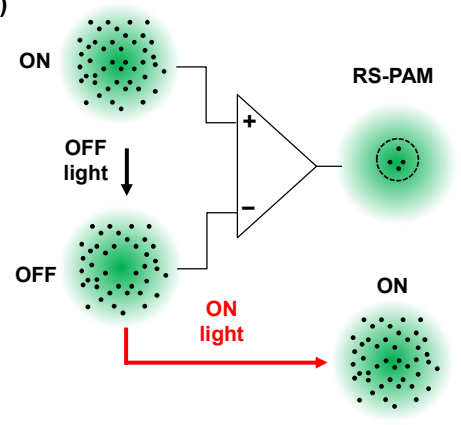

(b)

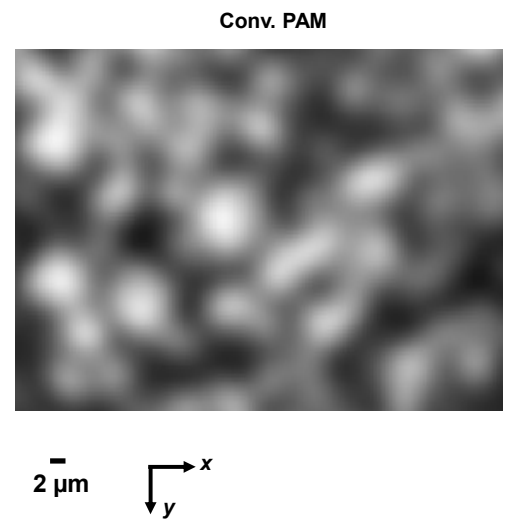

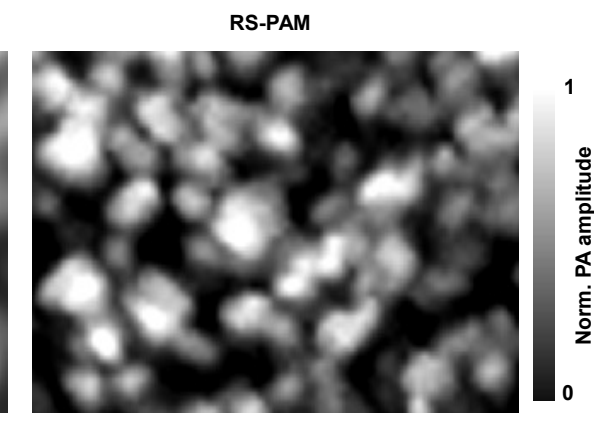

Figure 3. Spatial-resolution enhancement in RS-PAM. (a) Principle of RS-PAM with subdiffraction resolution. In the diffraction-limited excitation volume, some ON-state BphP1 molecules (dots) are switched to the OFF state, where the switching-off rate is proportional to the local excitation intensity. By fitting the nonlinear signal-decay dynamics, RS-PAM improves the spatial resolution in all dimensions. (b) Conventional (Conv.) PAM and RS-PAM images of BphP1-expressing bacteria densely fixed on a coverslip, showing the superior lateral resolution of RS-PAM. 
The lateral resolution enhancement of SPAM was demonstrated on a monolayer of BphP1-experessing bacteria densely growing on a cover glass. Both conventional PAM and SPAM imaging were performed with a single switching cycle. Compared with the conventional PAM image, the SPAM image shows superior lateral resolution, allowing better separation of closely packed bacteria (Figure 3b). The lateral resolution enhancement was estimated to be 1.7 folds [23]. We demonstrated the axial resolution enhancement of SPAM on multilayer of live BphP1-expressing U87 cells (sample thickness: $\sim 35 \mu \mathrm{m})$. For large targets, the conventional PAM relies on the poor axial resolution $(\sim 30 \mu \mathrm{m})$ provided by the time-resolved acoustic detection, and therefore was not able to resolve different layers of U87 cells. By contrast, SPAM was able to block the PA signals generated by out-of-focus cells and thus achieve optical sectioning $(\sim 0.4 \mu \mathrm{m})$. Cells at different depths were clearly resolved by SPAM. The axial resolution enhancement of SPAM is estimated to be $\sim 75$ folds.

\section{DISCUSSION}

In summary, we have demonstrated, for the first time, the combination of high-resolution deep-penetration PAT with the reversibly switchable non-fluorescent protein $\mathrm{BphP1}$, which can offer a valuable means for high-sensitivity imaging of genetically encoded biological processes at different length scales. With this new approach, we have achieved a PA detection sensitivity of $2.7 \mu \mathrm{M}$ of BphP1 or 20 mammalian cells at $\sim 10 \mathrm{~mm}$ depth, and have monitored the tumor growth and metastasis at the whole-body level in vivo. We also demonstrated PA imaging of BphP1 with optically-determined resolution in vivo and sub-diffraction PA imaging on bacteria and mammalian cells. The high-sensitivity BphP1-based imaging in this study has demonstrated the practicality of genetically encoded PA imaging, and thus opened the possibility for a wide range of biomedical applications. For example, transgenetic mouse modes that express BphP1 in the neuron cells can be used for longitudinal PA monitoring of neural network development, with high imaging sensitivity and specificity [24]. It also provides a new opportunity to study the dynamic interaction between neurons and its supporting vascular network.

\section{ACKNOWLEDGEMENTS}

This work was sponsored by US National Institutes of Health (NIH) grants DP1 EB016986 (NIH Director's Pioneer Award), R01 CA186567 (NIH Director's Transformative Research Award), 1S10 RR028864 and R01 CA159959.

\section{REFERENCES}

[1] Ntziachristos, V., "Going deeper than microscopy: the optical imaging frontier in biology," Nature Methods. 7(8), 603-614 (2010).

[2] Wang, L. H. V. and Hu, S., "Photoacoustic Tomography: In Vivo Imaging from Organelles to Organs," Science. 335(6075), 1458-1462 (2012).

[3] Zackrisson, S., van de Ven, S. M., and Gambhir, S. S., "Light in and sound out: emerging translational strategies for photoacoustic imaging," Cancer Res. 74(4), 979-1004 (2014).

[4] de la Zerda, A., et al., "Advanced contrast nanoagents for photoacoustic molecular imaging, cytometry, blood test and photothermal theranostics," Contrast Media \& Molecular Imaging. 6(5), 346-369 (2011).

[5] Luke, G. P., Yeager, D., and Emelianov, S. Y., "Biomedical Applications of Photoacoustic Imaging with Exogenous Contrast Agents," Annals of Biomedical Engineering. 40(2), $422-437$ (2012).

[6] Kircher, M. F., et al., "A brain tumor molecular imaging strategy using a new triple-modality MRIphotoacoustic-Raman nanoparticle," Nature Medicine. 18(5), 829-U235 (2012).

[7] Filonov, G. S., et al., "Deep-Tissue Photoacoustic Tomography of a Genetically Encoded Near-Infrared Fluorescent Probe," Angewandte Chemie-International Edition. 51(6), 1448-1451 (2012).

[8] Razansky, D., et al., "Multispectral opto-acoustic tomography of deep-seated fluorescent proteins in vivo," Nature Photonics. 3(7), 412-417 (2009). 
[9] Jathoul, A. P., et al., "Deep in vivo photoacoustic imaging of mammalian tissues using a tyrosinase-based genetic reporter," Nat Photon. advance online publication(2015).

[10] Krumholz, A., et al., "Photoacoustic microscopy of tyrosinase reporter gene in vivo," Journal of Biomedical Optics. 16(8), 080503 (2011).

[11] Laufer, J., et al., "In vitro characterization of genetically expressed absorbing proteins using photoacoustic spectroscopy," Biomedical Optics Express. 4(11), 2477-2490 (2013).

[12] Ntziachristos, V. and Razansky, D., "Molecular Imaging by Means of Multispectral Optoacoustic Tomography (MSOT)," Chemical Reviews. 110(5), 2783-2794 (2010).

[13] Shcherbakova, D. M., et al., "Natural Photoreceptors as a Source of Fluorescent Proteins, Biosensors, and Optogenetic Tools," Annual Review of Biochemistry. advance online publication(2014).

[14] Bellini, D. and Papiz, M. Z., "Structure of a Bacteriophytochrome and Light-Stimulated Protomer Swapping with a Gene Repressor," Structure. 20(8), 1436-1446 (2012).

[15] Li, H., et al., "Quaternary organization of a phytochrome dimer as revealed by cryoelectron microscopy," Proceedings of the National Academy of Sciences of the United States of America. 107(24), 10872-10877 (2010).

[16] Xia, J., et al., "Whole-body ring-shaped confocal photoacoustic computed tomography of small animals in vivo," Journal of Biomedical Optics. 17(5), 050506 (2012).

[17] Brakemann, T., et al., "A reversibly photoswitchable GFP-like protein with fluorescence excitation decoupled from switching," Nature Biotechnology. 29(10), 942-U132 (2011).

[18] Habuchi, S., et al., "Reversible single-molecule photoswitching in the GFP-like fluorescent protein Dronpa," Proceedings of the National Academy of Sciences of the United States of America. 102(27), 9511-9516 (2005).

[19] Gurskaya, N. G., et al., "Engineering of a monomeric green-to-red photoactivatable fluorescent protein induced by blue light," Nature Biotechnology. 24(4), 461-465 (2006).

[20] Lukyanov, K. A., et al., "Photoactivatable fluorescent proteins," Nature Reviews Molecular Cell Biology. 6(11), 885-891 (2005).

[21] Xia, J. and Wang, L. H. V., "Small-Animal Whole-Body Photoacoustic Tomography: A Review," Ieee Transactions on Biomedical Engineering. 61(5), 1380-1389 (2014).

[22] Yao, J. J., et al., "Photoimprint Photoacoustic Microscopy for Three-Dimensional Label-Free Subdiffraction Imaging," Physical Review Letters. 112(1), 014302 (2014).

[23] Yao, J. J., et al., "Reversibly switchable fluorescence microscopy with enhanced resolution and image contrast," Journal of Biomedical Optics. 19(8), 086018 (2014).

[24] Devor, A., et al., "The Challenge of Connecting the Dots in the BRAIN," Neuron. 80(2), 270-274 (2013).

[25] Chaffer, C. L. and Weinberg, R. A., "A Perspective on Cancer Cell Metastasis," Science. 331(6024), 15591564 (2011).

[26] Couzin-Frankel, J., "Cancer Immunotherapy," Science. 342(6165), 1432-1433 (2013). 